J. Austral. Math. Soc. (Series A) 45 (1988), 372-380

\title{
CHAIN CONDITIONS AND SEMIGROUP GRADED RINGS
}

\author{
E. JESPERS
}

(Received 7 October 1986; revised 26 October 1987)

Communicated by R. Lidl

\begin{abstract}
The following questions are studied: When is a semigroup graded ring left Noetherian, respectively semiprime left Goldie? Necessary sufficient conditions are proved for cancellative semigroup-graded subrings of rings weakly or strongly graded by a polycyclic-by-finite (unique product) group. For semigroup rings $R[S]$ we also give a solution to the problem in case $S$ is an inverse semigroup.
\end{abstract}

1980 Mathematics subject classification (Amer. Math. Soc.) (1985 Revision): 16 A 34, 16 A 03, 20 M 25.

\section{Introduction}

We are interested in the following questions: When is a semigroup graded ring (for example a semigroup ring) left Noetherian, respectively semiprime left Goldie? These turn out to be very difficult questions as they are even unsolved for group rings. However some results on Noetherianess are known if the ring is graded by a (polycyclic-by-) finite group (see [4], [16]). Therefore we are able to solve both questions (Section 1) for rings weakly or strongly graded by a cancellative monoid which is contained in a polycyclic-by-finite (unique product) group.

For non-cancellative semigroups the problems seem even more complicated. In Section 2 we give a solution for semigroup rings $R[S]$, where $S$ is an inverse semigroup.

(C) 1988 Australian Mathematical Society 0263-6115/88 $\$ A 2.00+0.00$ 


\section{Cancellative semigroup graded rings}

For the theory on semigroups and graded rings we refer to [3] and [12]. We briefly give some basic definitions and notation. Unless specifically stated, all rings are non-zero and all semigroups are non-zero.

Let $S$ be a semigroup. A ring $R$ is called an $S$-graded ring if there exists additive subgroups $R_{s}$ of $R$, indexed by the elements of $S$, such that $R=\bigoplus_{s \in S} R_{s}$ and $R_{s} R_{t} \subseteq R_{s t}$ for all $s, t \in S$. Moreover, if $l\left(R_{s}\right)=r\left(R_{s}\right)=0$ for all $s \in S$ then we call $R$ weakly graded. By $l(A)$, respectively $r(A)$, we denote the left (respectively right) annihilator of a subset $A$ in a ring $R$. An $S$-graded ring $R$ is called strongly graded if $R_{s} R_{t}=R_{s t}$ for all $s, t \in S$. Clearly every strongly group-graded ring with unity is weakly graded and it is easy to construct examples of weakly group graded rings which are not strongly graded. If $R$ is a ring and $S$ a semigroup then by $R[S]$ we denote the semigroup ring. If $S$ is cancellative and $R$ has a unity then $R[S]$ is a weakly $S$-graded ring.

Let $T$ be a subset of semigroup $S$ and let $R$ be an $S$-graded ring. By $R_{[T]}$ we denote $\bigoplus_{t \in T} R_{t}$. If $T$ is also a semigroup then $R_{[T]}$ is a $T$-graded ring, and we can also consider it as an $S$-graded ring.

A left ideal $L$ of an $S$-graded ring $R$ is called homogeneous if $L=\bigoplus_{s \in S} L_{s}$, where $L_{s}=R_{s} \cap L$. If $S$ is a monoid then its identity element is denoted by $e$. If $S$ is a semigroup then by $S^{1}$ we denote the smallest monoid containing $S$.

Let $R$ be a $G$-graded ring with unity, where $G$ is a group. In [9] we considered the left graded-maximal ring of quotients of $R$, denoted $Q_{\mathrm{gr}-\mathrm{Max}}^{l}(R)$, which is a $G$-graded ring and which is the graded version of the well known left maximal ring of quotients $Q_{\text {Max }}^{l}(R)$. We recall that

$$
Q_{\mathrm{gr}-\mathrm{Max}}^{l}(R)=\underset{\lim }{\longrightarrow} \operatorname{HOM}_{R}(L, R),
$$

that is, the direct limit of the system

$$
\begin{aligned}
& \left\{\operatorname{HOM}_{R}(L, R), \pi_{L, L^{\prime}}: \operatorname{HOM}_{R}(L, R) \rightarrow \operatorname{HOM}_{R}\left(L^{\prime}, R\right),\right. \\
& \left.L \supset L^{\prime}, L \text { and } L^{\prime} \text { are dense homogeneous left ideals of } R\right\} .
\end{aligned}
$$

Note that $\operatorname{HOM}_{R}(L, R)=\bigoplus_{h \in G} \operatorname{HOM}_{R}(L, R)_{h}$ : where $\operatorname{HOM}_{R}(L, R)_{h}$ is the additive abelian group of all $R$-linear maps $f: L \rightarrow R$ which are graded morphisms of degree $h$, that is $\left(L_{g}\right) f \subseteq R_{g h}$ for all $g \in G$. A left ideal $L$ of $R$ is dense if and only if for all $x \in R$ we have $r\{y \in R \mid y x \in L\}=\{0\}$. The following characterization for $Q_{\mathrm{gr}-\mathrm{Max}}^{l}(R)$ can be found in [9].

PROPOSITION 1.1. Let $G$ be a group and $R$ and a $G$-graded ring with unity. Then $Q=Q_{\mathrm{gr}-\operatorname{Max}}^{l}(R)$ is uniquely determined (withing the category of graded rings) by the following four properties:

(1) $R$ is a graded subring of $Q$, that is, $R_{g} \subseteq Q_{g}$ for all $g \in G$; 
(2) for every $q \in Q$ there exists a dense homogeneous left ideal $L$ of $R$ such that $L q \subseteq R$;

(3) if $q \in Q$ and $L q=0$ for some dense homogeneous left ideal of $R$, then $q=0$

(4) if $f \in \operatorname{HOM}_{R}(L, R), L$ a dense homogeneous left ideal of $R$, then there exists $q \in Q$ with $x f=x q$ for all $x \in L$.

Hence it follows that

$$
Q_{\mathrm{Max}}^{l}\left(Q_{\mathrm{gr}-\mathrm{Max}}^{l}(R)\right)=Q_{\mathrm{Max}}^{l}(R) .
$$

And thus, if $R$ is trivially graded $Q_{\mathrm{Max}}^{l}\left(Q_{\mathrm{Max}}^{l}(R)\right)=Q_{\mathrm{Max}}^{l}(R)$. Moreover, if $R$ is weakly G-graded then

$$
Q_{\mathrm{Max}}^{l}\left(R_{e}\right)=\left(Q_{\mathrm{gr}-\mathrm{Max}}^{l}(R)\right)_{e} .
$$

PROPOSITION 1.2. Let $R$ be a weakly $G$-graded ring with unity, where $G$ is a group. If $S$ is a submonoid of $G$ such that $G$ is the group of left quotients of $S$, then

$$
Q_{\mathrm{gr}-\mathrm{Max}}^{l}(R)=Q_{\mathrm{gr}-\mathrm{Max}}^{l}\left(R_{[S]}\right) .
$$

For this we consider $R_{[S]}$ as a $G$-graded ring.

ProOF. This result has been proved in [9], using Proposition 1.1. But here is a short direct proof. For any $t=s^{-1} s_{1} \in G, s, s_{1} \in S$, and any $b \in R_{t}$ consider the graded homomorphism $f_{b}: R_{[S]} R_{s} \rightarrow R_{[S]} R_{s_{1}}$ give by $f_{b}(x)=x b$. Then the rule $b \rightarrow f_{b}$ defines an embedding $R \rightarrow Q_{\mathrm{gr}-\mathrm{Max}}^{l}\left(R_{[S]}\right)$ and the result follows.

LEMMA 1.3. Let $R$ be a semiprime ring with unity. Then $R$ is left Goldie if and only if $Q_{\mathrm{Max}}^{l}(R)$ is left Goldie.

ProOF. This result is well known; see [17].

LEMMA 1.4. Let $S$ be a cancellative semigroup and let $R$ be an $S$-graded ring with $R_{s} \neq 0$ for all $s \in S$. If $R_{[S]}$ is left Goldie then $S$ has a group of left quotients.

ProOF. We show that $S$ satisfies the left Ore condition. The idea of the proof is well known, see [5]. Suppose $S s \cap S t=\varnothing, s, t \in S$. We claim that $\bigcup_{i=0}^{\infty} S s t^{i}$ is a disjoint union of left ideals of $S$. Indeed, suppose $a \in S s t^{i} \cap S s t^{j}, i<j$; that is, $a x s t^{i}=y s t^{j}$ for some $x, y \in S$. It follows that $x s=y s t^{j-i} \in S s \cap S t$, a contradiction. Therefore $\sum_{i=0}^{\infty} R_{\left[S s t^{i}\right]}$ is a direct sum of left ideals of $R_{[S]}$, a contradiction. Hence the result follows.

If $R$ is a ring we denote by $J(R)$ the Jacobson radical of $R$. 
Proposition 1.5. Let $G$ be a group with submonoid $S \neq\{e\}$, and let $R$ be a weakly $G$-graded ring with unity such that $R_{e}$ is semiprime. If $R[S]$ is left Goldie then $R_{e}$ is left Goldie and $S$ has a group of left quotients. If, moreover, $G$ is polycyclic-by-finite then $J\left(R_{[S]}\right)$ is nilpotent.

PROOF. If $L$ is a left ideal of $R_{e}$ then $R L$ is a homogeneous left ideal of $R$ such that $\left(R_{[S]} L\right)_{e}=L$. It is then easy to check that $R_{[S]}$ being left Goldie implies $R_{e}$ is left Goldie. Lemma 1.4 yields that $S$ has a group of left quotients.

If $G$ is polycyclic-by-finite, $G$ has a normal subgroup $N$ of finite index which is poly-infinite cyclic, in particular $N$ is a unique product (u.p.) group (see [15]). One can easily adapt the proof of Theorem 1.2 of [8] to show that this theorem remains valid under our assumptions. Therefore $J\left(R_{[S \cap N]}\right)=0$. Now $R_{[S]}$ has a natural $G / N$ gradation; the component of degree $e$ being $R_{[S \cap N]}$. Lemma 2.1 in [10] implies that $J\left(R_{[S]}\right)$ is a nil ideal, and hence by Lanski's Theorem [11], $J\left(R_{[S]}\right)$ is nilpotent.

If in the previous theorem the group $G$ is also u.p. then the converse of the theorem is also true.

THEOREM 1.6. Let $G$ be a polycyclic-by-finite u.p. group with submonoid $S \neq\{e\}$, and let $R$ be a weakly $G$-graded ring with unity. Then $R_{[S]}$ is semiprime left Goldie if and only if $R_{e}$ is semiprime left Goldie. Moreover, in this case, $J\left(R_{[S]}\right)=0$.

ProOF. As mentioned in the previous proof, under our assumption Theorem 1.2 of [8] remains valid. Hence, if $R_{e}$ is left Goldie then, $R_{e}$ is semiprime if and only if $R_{[S]}$ is semiprime, and $J\left(R_{[S]}\right)=0$ in this case. Hence Proposition 1.5 yields that $R_{e}$ is semi-prime left Goldie if $R_{[S]}$ is semiprime left Goldie.

We now prove the converse. Since $R_{[S]}$ is semiprime if follows from Proposition 1.1, Proposition 1.2 and Lemma 1.3 that it is sufficient to show that $Q=Q_{\mathrm{gr}-\mathrm{Max}}^{l}(R)$ is left Goldie. Again by Proposition 1.1, $Q_{e}=Q_{\mathrm{Max}}^{l}\left(R_{e}\right)$. Since $Q_{\mathrm{Max}}^{l}\left(R_{e}\right)=Q_{c l}^{l}\left(R_{e}\right), Q_{e}$ is a left Noetherian ring. Hence, by Quinn's results [16], $Q$ is left Noetherian; and thus left Goldie.

EXAMPLE. The following example, which can be found in [17], shows that if $R_{[S]}$ is not contained in a weakly $G$-graded ring then Theorem 1.6 does not necessarily hold. Let $K$ be a field and $\phi$ a monomorphism of $K$ which is not bijective. Let $R=K[X, \phi]$, the skew polynomial ring. Let $k \in K$ such that $k \notin \phi(K)$, then $K[X, \phi] X k \cap K[X, \phi] X=0$. Hence $R$ is a domain which does not satisfy the left Ore condition. Therefore $R$ is not left Noetherian. One easily verifies that $R$ is not contained in a weakly group graded ring. 
COROLLARY 1.7. Let $R$ be a ring with unity and $\{e\} \neq S$ a cancellative monoid. If $R[S]$ is semiprime left Goldie then $R$ is semiprime left Goldie and $S$ has a group of left quotients, say $G$. If $G$ is polycyclic-by-finite and u.p. then the converse holds; if $G$ is polycyclic-by-finite then the necessary conditions imply that $J(R[S])$ is nilpotent.

Proof. Obviously $R$ is semiprime if $R[S]$ is semiprime. Note also that if $S$ has $G$ as group of left quotients then the group ring $R[G]$ is weakly $G$-graded and $R[S]=(R[G])_{[S]}$. The result follows from Lemma 1.4 and Proposition 1.5 and Theorem 1.6. In the remainder of this section we are interested in (left) Noetherian graded rings. We first state a very well known result (see [1]).

LEMMA 1.8. Let $P$ be a partially ordered set for the relation $\leq$. Suppose that the following conditions are satisfied:

(1) ascending chain condition (a.c.c.), that is, $P$ does not contain an infinite ascending chain $s_{1}<s_{2}<s_{3}<\cdots$;

(2) descending chain condition (d.c.c.), that is, $P$ does not contain an infinite descending chain $s_{1}>s_{2}>s_{2}, \ldots$;

(3) $P$ does not contain an infinite set of incomparable elements, that is, a subset $A$ of $P$ is said to consist of incomparable elements if for all $a, b \in A, a \npreceq b$ and $b \$ a$.

Then $P$ is a finite set.

A graded ring is said to be left gr-Noetherian if it satisfies the ascending chain condition on homogeneous left ideals. A semigroup $S$ is said to be (left) Noetherian if it satisfies the ascending chain condition on (left) ideals. Note that this definition does not agree with the usual definition in the literature (see [7]).

LEMMA 1.9. Let $S$ be an arbitrary semigroup and let $R$ be an $S$-graded ring such that $R_{s} \neq 0$ for all $s \in S$. If $R$ is left gr-Noetherian then $S$ is left Noetherian. If, moreover, $S$ is a monoid then $R_{e}$ is left Noetherian.

ProoF. If $A$ is a left ideal of $S$ then $R_{[A]}$ is a left ideal of $R$. If $e \in S$ and $L_{e}$ is a left ideal of $R_{e}$, then $R L_{e}$ is a left ideal of $R$ with $R L_{e} \cap R_{e}=L_{e}$. Because of the assumption on $R$ the result now follows easily.

The converse of the previous lemma is more of an interest.

Proposition 1.10. Let $G$ be a group and let $S$ be a left Noetherian submonoid. If $R$ is a strongly $G$-graded ring with unity and such that $R_{e}$ is left Noetherian then $R_{[S]}$ is left gr-Noetherian. 
Proof. Let $J=\sum_{s \in S} J_{s}$ be an homogeneous left ideal of $R_{[S]}$. For every $s \in S$, denote $I_{s}=R_{s^{-1}} J_{s}$. We claim that $L=\left\{I_{s} \mid s \in S\right\}$ is a finite set. Clearly $L$ is a partially ordered set for the inclusion relation. To prove that $L$ is finite we check the conditions (1), (2) and (3) of Lemma 1.8.

(1) $L$ has no infinite ascending chain, because all elements of $L$ are left ideals in $R_{e}$ and because $R_{e}$ is left Noetherian.

(2) Suppose $I_{s_{1}} \supsetneq I_{s_{2}} \supsetneq I_{s_{3}} \subsetneq \cdots$ is an infinite descending chain in $L$. For every $n \in \mathrm{N}_{0}$, let $A_{n}=\left\{s \in S \mid I_{s_{n}} \subseteq I_{s}\right\}$. Then $A_{1} \subsetneq A_{2} \subsetneq A_{3} \subsetneq \cdots$. If we prove that every $A_{n}$ is a left ideal of $S$, then we are in contradiction with the assumption on $S$; and hence $L$ satisfies d.c.c. Thus fix $n \in \mathrm{N}_{0}$ and we prove that $A_{n}$ is a left ideal of $S$. For this let $s \in A_{n}$, that is, $I_{s_{n}} \subseteq I_{s}$, and let $t \in S$. Then

$$
I_{s}=R_{s^{-1}} J_{s}=R_{s^{-1}} R_{t^{-1}} R_{t} J_{s}=R_{(t s)^{-1}} R_{t} J_{s} \subseteq R_{(t s)^{-1}} J_{t s}=I_{t s},
$$

hence $I_{s} \subseteq I_{t s}$. Hence $I_{s_{n}} \subseteq I_{t s}$, that is, $t s \in A_{n}$. Therefore $A_{n}$ is a left ideal of $S$.

(3) Suppose $\left\{I_{s_{i}} \mid i \in \mathbf{N}_{0}\right\}$ is an infinite set of incomparable elements of $L$. Then, for every $n \in \mathrm{N}_{0}$, let $B_{n}=\left\{s \in S \mid I_{s_{i}} \subset I_{s}\right.$ for some $\left.1 \leq i \leq n\right\}$. With the notations as in (2), $B_{n}=A_{1} \cup \cdots \cup A_{n}$. Hence $B_{n}$ is a left ideal of $S$. Clearly $B_{1} \subsetneq B_{2} \subsetneq B_{3} \subsetneq \cdots$. This is again in contradiction with the left Noetherianess of $S$.

So, let $\left\{I_{s_{1}}, \ldots, I_{s_{n}}\right\}$ be all non zero elements of $L$. With notations as in (2), for each $1 \leq i \leq n, A_{i}$ is a left ideal of $S$. Hence $A_{i}=\bigcup_{j=1}^{n_{i}} S s_{i, j}$ and $I_{s_{i}}=\sum_{j^{\prime}=1}^{m_{i}} R_{e} x_{i, j^{\prime}}$. Now because $R$ is strongly graded, each $R_{s_{i, j}}$ is finitely generated as a left $R_{e}$-module. Let $R_{s_{i, j}}=\sum_{k=1}^{(i, j)} R_{e} r_{s_{i, j}}^{(k)}$. We claim that $J$ is generated as a left $R_{[S]^{-i d e a l}}$ by the finitely many elements $r_{s_{i, j}^{(k)}} x_{i, j^{\prime}}, 1 \leq$ $i \leq n, 1 \leq j \leq n_{i}, 1 \leq j^{\prime} \leq m_{i}, i \leq k \leq l_{(i, j)}$. To prove this let $s \in S$ be such that $J_{s} \neq 0$. Then, $J_{s}=R_{e} J_{s}=R_{s} I_{s}$ (note that because $e \in S, J_{s}$ is a left $R_{e}$-module). Suppose $I_{s}=I_{s_{i}}$. Then $s \in A_{i}$ and thus $s=t s_{i, j}$ for some $j$. Hence $I_{s_{i}, j} \subseteq I_{s}$. Since $s_{i, j} \in A_{i}$ it follows that $I_{s_{i}} \subseteq I_{s_{i}, j} \subseteq I_{s}$. Thus $I_{s_{i}}=I_{s_{i, j}}=I_{s}$. It follows that $J_{s}=R_{s} I_{s}=R_{s} I_{s_{i}}=R_{t} R_{s_{i, j}} I_{s_{i, j}}=$ $R_{t} \sum_{j^{\prime}=1}^{m_{j}} R_{s_{i, j^{\prime}}} x_{i, j^{\prime}}=R_{t} \sum_{j^{\prime}=1}^{m_{i}} \sum_{j^{\prime}=1}^{l_{(i, j)}} R_{e} r_{s_{i, j}}^{(k)} x_{i, j^{\prime}}$. Thus $J_{s} \subseteq \sum R_{[S]} r_{s_{i, j}}^{(k)} x_{i, j^{\prime}}$. Because $I_{s} \stackrel{=}{=} I_{s_{i}}=I_{s_{i, j}}$ it follows that (as in (2)) $R_{s_{i, j}} I_{s_{i}}=R_{s_{i, j}} I_{s_{i, j}} \subseteq J$. Hence all $r_{r_{i, j}}^{(k)} x_{i, j}$, belong to $J$. The result follows.

Note that in the above proof the assumptions that $S \subseteq G$ and that $R$ is strongly $G$-graded are used only (1) to transform $J_{s}$ to $I_{s}$ and vice versa and (2) to obtain that every $R_{s}$ is a finitely generated left $R_{e}$-module. Now (1) and (2) are automatically satisfied if we are interested in semigroup rings of arbitrary monoids. For this reason and because of Lemma 1.9 we can state 
PROPOSITION 1.11. Let $R$ be a ring with unity and $S$ a monoid. Then $R[S]$ is left gr-Noetherian if and only if $R$ and $S$ are left Noetherian.

In [7] it is shown that Proposition 1.11 is not longer true (even for $S$ commutative and cancellative) if $S$ does not contain an identity.

The following corollary shows that Proposition 1.11 remains valid for certain strongly graded rings.

COROLLARY 1.12. Let $S$ be a submonoid of a polycyclic-by-finite group $G$, and let $R$ be a strongly $G$-graded ring with unity. Then $R_{[S]}$ is left Noetherian if and only if $R_{e}$ and $S$ are left Noetherian.

PROOF. Assume that $R_{[S]}$ is left Noetherian. Obviously $R_{[S]}$ is then left gr-Noetherian. Hence Lemma 1.9 implies that $R_{e}$ and $S$ are left Noetherian. Conversely, by Proposition 1.10, $R_{[S]}$ is left gr-Noetherian. Now, considering $R_{[S]}$ as a $G$-graded ring the result follows from [16] because $G$ is polycyclic-byfinite.

Note that if in the above corollary $R_{[S]}$ is not contained in a strongly graded ring, then the result is no longer true because of the example after Theorem 1.6. In the case of left Noetherian semigroup rings $R_{e}[S]$ of cancellative semigroups it follows that $R_{e}[S]$ is contained in a strongly group graded ring $R$ such that $R_{e}[S]=R_{[S]}$. Indeed because of Lemma $1.9 S$ is left Noetherian and hence has a group $G$ of left quotients. The group $\operatorname{ring} R=R_{e}[G]$ satisfies the conditions.

\section{Rings graded by a subsemigroup of an inverse semigroup}

In this section we study when semigroup rings of inverse semigroups are semiprime left Goldie. To do so we first prove two results on rings graded by a semilattice. A semilattice is a commutative semigroup consisting of idempotents. It has an ordered structed $\leq$ defined by $s \leq t$ if and only if $s=s t$.

LEMMA 2.1. Let $R$ be an $S$-graded ring, $S$ a semigroup with $E(S)$ a commutative subsemigroup of idempotents of $S$. Suppose that for all $s, t \in E(S), R_{s}$ has a unity $u_{s}$ and $u_{s} u_{t}=u_{s t}$. If $R$ is left Goldie then $E(S)$ is finite.

ProOF. $\left\{u_{s} \mid s \in E(S)\right\}$ is a set of commuting idempotents of $R$. So it must be finite, because otherwise $R$ has an infinite set of orthogonal idempotents (see [6], Chapter 3, Ex. 14). 
PROPOSITION 2.2. With notations and assumptions as in Lemma 2.1. Moreover, assume $S=E(S)$. Then $R$ is left Goldie if and only if $S$ is finite and each $R_{s}, s \in S$, is left Goldie.

Proof. Assume $S$ is finite. Let $f$ be the least element of $S$. Since $R_{f}$ has an identity and is an ideal of $R, R \cong R_{f} \oplus R / R_{f}$. Similarly $R \cong R_{f} \oplus R_{g} \oplus$ $R /\left(R_{f} \oplus R_{g}\right)$ for a minimal nonzero $g \in S$. By an induction $R \cong \bigoplus_{s \in S} R_{s}$ (note that $R /\left(R_{f} \oplus R_{g}\right)$ is $S /\{f, g\}$-graded. Using Lemma 2.1 the result follows

THEOREM 2.3. Let $R$ be a ring with unity and $S$ an inverse semigroup. Then $R[S]$ is semiprime left Goldie if and only if $E(S)$ is finite and, for every maximal subgroup $G$ of $S, R[G]$ is semiprime left Goldie. If $R[S]$ is prime left Goldie, then $S$ is a group. $E(S)$ is the set of idempotents of $S$.

ProOF. Assume that $E(S)$ is finite. In this case (see for example [10]) it follows that

$$
R[S] \cong \bigoplus_{k=0}^{n} M_{n_{k}}\left(R\left[G_{k}\right]\right),
$$

where the $G_{k}$ are the maximal subgroups of $S$, and $n_{0}=1$. In particular $G_{0}$ is an ideal of $S$ and $R\left[G_{0}\right]$ is a direct summand of $R[S]$.

Hence $R[S]$ is semigroup left Goldie if and only if each $M_{n_{k}}\left(R\left[G_{k}\right]\right)$ is semiprime left Goldie, and this is equivalent to $R\left[G_{k}\right]$ being semiprime left Goldie. The result now follows from Lemma 2.1 and Proposition 2.2.

If $R[S]$ is prime left Goldie then it follows from the above direct sum that $S=G_{0}$, that is, $S$ is a group.

J. Kerr (see for example [2]) has given an example of a commutative Goldie ring $R$ such that $M_{2}(R)$ is not a Goldie ring. It is then clear from the above proof that the previous theorem no longer holds without the assumption that $R[S]$ is semiprime.

A similar proof as that of Theorem 2.3 shows (see [18]) that $R[S]$ is left Noetherian if and only if $E(S)$ is finite and $R[G]$ is left Noetherian for every maximal subgroup $G$ of $S$ ( $S$ inverse semigroup).

To finish we mention the referee pointed out to the author that J. Okinski, in a recent paper [14], proved the following nice result. If the semigroup ring $R[S], S$ an arbitrary semigroup, is left and right Noetherian, then $S$ is finitely generated. As was done in an earlier version of this paper, for cancellative semigroups and subsemigroups of a semilattice of groups this can easily be proved using the previous results. 


\section{Acknowledgement}

The author wishes to thank the referee for several valuable corrections and improvements.

\section{References}

[1] G. Birkhoff, Lattice theory Amer. Math. Soc. Colloq. Publ., vol. 25, Amer. Math. Soc., Providence, R.I., 1979.

[2] A. Chatters and C. R. Hajarnavis, Rings with chain conditions (Pitman, 1908).

[3] A. H. Clifford and G. B. Preston, The algebraic theory of semigroups, Vol. I (Math. Surveys, Amer. Math. Soc., no. 7, Providence, R.I., 1961).

[4] M. Cohen and L. H. Rowen, 'Group graded rings', Comm. Algebra 11 (11) (1983), 12531270.

[5] P. M. Cohn, Algebra II (John Wiley and Sons, London, 1977).

[6] C. Faith, Algebra II (Springer-Verlag, Berlin, 1973).

[7] R. Gilmer, Commutative semigroup rings, (Univ. Chicago Press, Chicago, 1984).

[8] E. Jespers, 'On radicals of graded rings and applications to semigroup rings', Comm. Algebra 13 (11) (1985), 2457-2472.

[9] E. Jespers and P. Wauters, 'A general notion of noncommutative Krull rings', J. Algebra 112 (2) (1988), 388-415.

[10] I. B. Kozuhov, 'Self-injective semigroup algebras of inverse semigroups', Izv. Vyss. Ucebn. Zaved. 2 (1981), 46-51.

[11] C. Lanski, 'Nil subrings of Goldie rings are nilpotent', Canad. J. Math. 21 (1969), 904-907.

[12] C. Nastasescu and F. Van Oystaeyen, Graded ring theory, (North-Holland, Amsterdam, 1982).

[13] J. Okninski, 'On the radical of semigroup algebras satisfying polynomial identities', $M a t h$. Proc. Cambridge Philos. Soc. 99 (1986), 45-50.

[14] J. Okninski,'Noetherian property for semigroups rings', Proc. Int. Conf. on Ring Theory, Granada, 1986, to appear.

[15] D. S. Passman, The algebraic structure of group rings, (John Wiley and Sons, New York, 1977).

[16] D. Quinn, 'Group-graded rings and duality', Trans. Amer. Math. Soc. 292 (1985), 155-168.

(17) B. Stenström, Rings of quotients, (Springer-Verlag, Berlin, 1975).

[18] P. Wauters and E. Jespers, 'Rings graded by an inverse semigroup with only finitely many idempotents', Houston J. Math., to appear.

Department of Mathematics

Memorial University of Newfoundland

St. John's, Newfoundland

Canada A1C 5S7 